\title{
Analysis of HIV testing refusal among patients aged less than 21 years in the Pediatric Emergency Department
}

This article was published in the following Dove Press journal: HIVIAIDS - Research and Palliative Care

\author{
Kenneth Soyemi ${ }^{1,2}$ \\ Teddy Muisyo ${ }^{1,3}$ \\ Yaa KariKari ${ }^{1,4}$ \\ Kun-UK David Lee ${ }^{5}$ \\ Peter Nguyen' \\ Karen E Simpson' \\ Kevin Regis ${ }^{2}$ \\ Lisa Henry Reid'
}

'Department of Pediatrics, Cook County Health and Hospitals System, John H Stroger Jr Hospital, Chicago, IL, USA; ${ }^{2}$ Department of Emergency Medicine, Cook County Health and Hospitals System, John H Stroger Jr Hospital, Chicago, IL, USA; ${ }^{3}$ Division of Critical Care, Department of Pediatrics, Washington University School of Medicine, St Louis, MO, USA; ${ }^{4}$ Department of Pediatrics, Division of Cardiology, Advocate Christ Hospital, Chicago, IL, USA; ${ }^{5}$ School of Medicine, Rosalind Franklin Medical School, North Chicago, IL, USA
Correspondence: Kenneth Soyemi Department of Pediatrics and Emergency Medicine, Cook County Health and Hospitals System, John H Stroger Jr Hospital, 1901 West Harrison Street, Chicago, IL 606I2, USA

Email ksoyemi@cookcountyhhs.org

\begin{abstract}
HIV testing in the Pediatric Emergency Department (PED) is a novel concept as adolescents, and young adults, use the PED as point of care or first point of contact with the health care system. Our objective was to study the HIV nontesting data and factors that influenced testing decision among patients receiving care in our PED. We designed a survey that inquired about testing acceptance, reasons for rejection, satisfaction with testing conditions, and understanding of the consequence of HIV test results. We approached 500 patients across all shifts in the PED; for analysis, categorical variables were created using demographic data (race, age, ethnicity, marital status, level of education). Forward conditional binary logistic regression was used to explore the effect of various independent predictors on HIV testing rejection with the strength of association measured with adjusted odds ratio (OR), and their $95 \%$ CIs. We conducted model fitting by plotting residuals, Hosmer and Lemeshow test statistic, and area under the curve completed using predicted probabilities. We used SPSS Version $25^{\mathrm{TM}}$, Microsoft Excel $2016^{\mathrm{TM}}$ for data preparation and analysis. Of the 500 patients approached, $423(84.6 \%)$ completed the survey, median (interquartile) age of survey participants was 19 (17-20) years, $158(37.4 \%)$ rejected HIV testing, $284(67.1 \%)$ were older than 18 years of age, $200(47.3 \%)$ were males, $154(36.4 \%)$ were white, and 127 (30\%) were of Hispanic origin. The most common reason for rejecting HIV was low risk perception declared by $79(50 \%)$ respondents. In multivariate analysis, age $<18$ years (OR, 3.5; 95\% CI, 2.3-5.5, $P<0.00$ ) and being Hispanic (OR, 2.5; 95\% CI, 1.6-3.8, $P<0.00$ ) were significant predictors for respondent nontesting. Hosmer and Lemeshow test was not significant, $P=0.42$, and area under the curve was 0.67 (95\% CI, 0.61-0.76). Respondents, $<18$ years were more likely to reject HIV testing because of low perception of risk. Program addressing risk perception which emphasizes safe health practices should be developed to reduce HIV transmission.
\end{abstract}

Keywords: HIV test, adolescent, testing refusal, emergency department

\section{Brief report}

HIV testing in the Pediatric Emergency Department (PED) is a novel concept as adolescents, and young adults, use the PED as a point of care or first point of contact with the health care system. Our study objective was to describe the reasons why patients seen in the PED refused HIV testing. The Centers for Disease Control and Prevention (CDC) recommended an opt-out approach to HIV testing, which excludes the pretest counseling and written consent which were often previously cited as barriers to routine HIV testing. ${ }^{1,2}$ Routine screening in the PED and other health care setting point of care (POC) such as urgent care, walk-in clinics takes away the stigma of dedicated HIV testing centers. POCs are convenient and discreet; the casual attitude to HIV testing is also often touted as a reason for testing acceptance. ${ }^{3}$ The study was 
approved by the John H Stroger Hospital Institutional Review Board, and consent waived because the research (being a survey) involved less than minimal risk to the patients and the waiver did not adversely affect the rights and welfare of the patients. The survey inquired about testing acceptance, reasons for nonacceptance, satisfaction with testing conditions, and understanding of the consequence of HIV test results. We approached 500 patients across all shifts in the PED from April 2016 through December 2017. Inclusion criteria were patients who were aged 13-21 years seeking care in the PED, and who had not had an HIV test recorded in our electronic medical records in the preceding 2 years; participation was voluntary.

For analysis, categorical variables were created using demographic data (race, age, ethnicity, marital status, level of education). Bivariate analysis and forward conditional binary logistic regression was used to explore the effect of various independent predictors on HIV testing nonacceptance with the strength of association measured with crude odds ratio and adjusted odds ratio (AOR), and their 95\% CIs. We conducted model fitting by plotting residuals, Hosmer and Lemeshow test statistic ( $\mathrm{H}-\mathrm{L}$ test), and area under the curve (AUC) completed using predicted probabilities. We used SPSS Version $25^{\mathrm{TM}}$ (IBM Corporation, Armonk, NY, USA), Microsoft Excel 2016 ${ }^{\mathrm{TM}}$ (Microsoft Corporation, Redmond, WA, USA) for data preparation and analysis.

Of the 500 patients approached $423(84.6 \%, 95 \% \mathrm{CI}$, $81.2 \%-87.6 \%)$ completed the survey, and 77 (15.4\%, 95\% CI, 12.4\%-18.8\%) either declined participation or did not complete the survey. Median (interquartile) age of survey participants was 19 (17-20) years; of the 423 participants, 158 (37.4\%, 95\% CI, 32.4\%-42.0\%) declined HIV testing,
$284(67.1 \%, 95 \%$ CI, $62.6 \%-71.5 \%)$ were aged $>18$ years of age, $200(47.3 \%$, 95\% CI, 42.6\%-52.1\%) were males, 154 (36.4\%, 95\% CI, 31.9\%-41.1\%) were white, and $127(30 \%$, $95 \%$ CI, $25.7 \%-34.6 \%$ ) were of Hispanic origin. The majority of those that rejected testing were heterosexual (154 of 158); we stratified testing rejection data into two subgroups (patients aged $<18$ years $[78,49.4 \%]$ compared with patients older than 18 years [80, 50.6\%]). The most common reason for rejecting HIV testing was a low risk perception declared by $78(49.4 \%)$ of 158 respondents; the health care provider not asking the patient accounted for 56 (35.4\%) cases, and receipt of a recent test somewhere else was reported by 38 $(24 \%)$ cases. Additional bivariate analysis using the previously described age group category demonstrated that younger age persons had three times the odds of refusing the testing due to low risk perception, and persons older than 18 years refused to test because of a recent test (Table 1). In multivariate analysis, age $<18$ years (AOR, 3.5; 95\% CI, 2.3-5.5 $P<0.00$ ) and being Hispanic (AOR, 2.5; 95\% CI, 1.6-3.8, $P<0.00)$, were significant predictors for rejecting HIV testing (Table 2). $\mathrm{H}-\mathrm{L}$ test was not significant, $P=0.42$, and AUC was 0.67 (95\% CI, 0.61-0.76). Modeling analysis demonstrated that the constant only model was statistically significant, indicating that the predictors reliably distinguished between patients who rejected testing and those that accepted testing. The nonsignificant $\mathrm{H}-\mathrm{L}$ test means implies that the model's estimates fit the data at an acceptable level and that the model prediction does not significantly differ from what we observed. AUC measures discrimination, that is, the ability of the model to correctly classify patients that rejected HIV test and those that accepted testing. AUC in this instance was $70 \%$, making it a good model (Figure 1). Among teenagers

Table I Analysis of reasons for not testing stratified by age group

\begin{tabular}{|c|c|c|c|c|c|c|}
\hline Reason for HIV testing rejection & $\begin{array}{l}\text { Participants, } \\
\mathrm{N}=158\end{array}$ & $\begin{array}{l}\text { Less than } 18 \\
\text { years }(\mathrm{N}=78)\end{array}$ & $\begin{array}{l}\text { Older than } 18 \\
\text { years }(\mathrm{N}=80)\end{array}$ & $P$-value & OR & $95 \% \mathrm{Cl}$ \\
\hline I don't think I am at risk for HIV infection (N, \%) & $79(50.0)$ & $50(64.0)$ & $29(36.0)$ & 0.00 & 3.14 & $1.64-6.01$ \\
\hline No one asked me about it (N, \%) & $56(35.4)$ & $25(32.0)$ & $31(38.0)$ & 0.50 & 0.76 & $0.39-1.46$ \\
\hline I was recently tested for HIV (N, \%) & $38(24.0)$ & $06(8.0)$ & $32(40.0)$ & 0.00 & 0.12 & $0.04-0.32$ \\
\hline I don't want to be tested at this center $(\mathrm{N}, \%)$ & $08(5.0)$ & $04(5.1)$ & $04(5.0)$ & 1.00 & 1.02 & $0.24-4.25$ \\
\hline
\end{tabular}

Abbreviation: OR, odds ratio.

Table 2 Predictors of HIV declination testing

\begin{tabular}{|l|l|l|l|l|}
\hline Factor & Tested $\mathbf{( N = 2 6 5 )}$ & Did not test $(\mathbf{N}=\mathbf{1 5 8})$ & Crude OR (95\% Cl) & AOR (95\% Cl) \\
\hline Hispanic (N, \%) & $63(23)$ & $64(40)$ & $2.2(1.3-2.0)$ & $2.5(1.6-3.8)$ \\
\hline$<18$ years (N, \%) & $61(23)$ & $78(49)$ & $3.3(2.1-4.9)$ & $3.5(2.3-5.5)$ \\
\hline
\end{tabular}

Abbreviations: AOR, adjusted odds ratio; OR, odds ratio. 


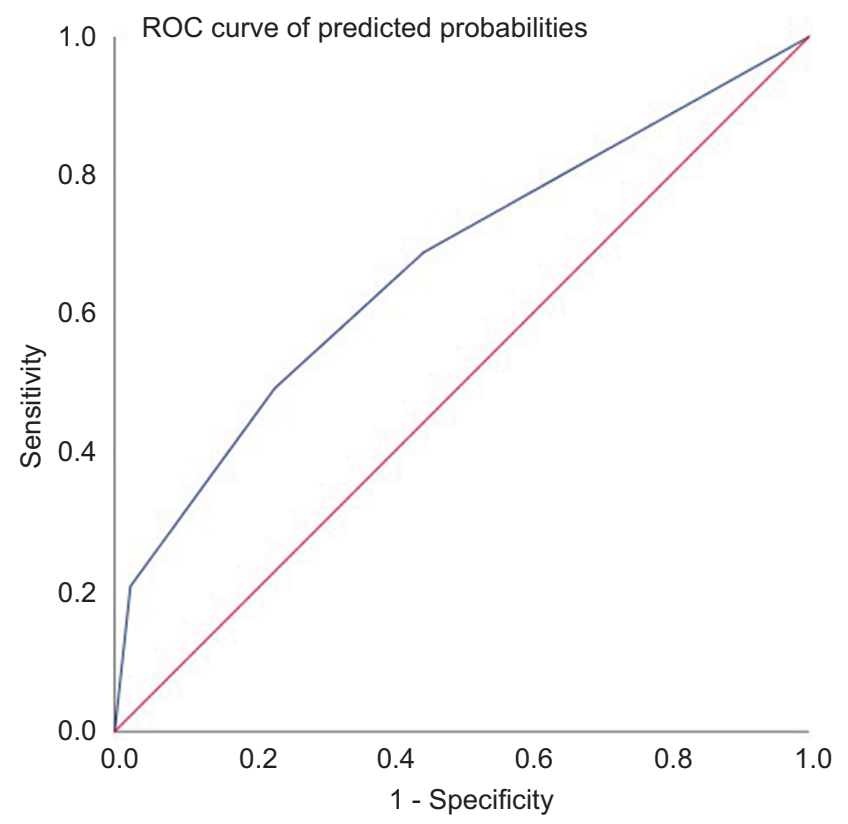

Figure I Model ROC.

Abbreviation: ROC, receiver operating characteristic.

and young adults, the PED is often the only POC they might have with a health care professional in years. One of the main barriers is physicians are not testing eligible patients visiting the ED. ${ }^{3-5}$ Physician recommendation to patients to get tested for HIV has been found to be a significant determinant of patient testing behaviors, and thus the low testing percentage can be directly attributed to physician's decisions not to test for HIV., ${ }^{4,5}$ Therefore, changing physician behavior enabling them to offer the HIV test to all the eligible patients will lead to an improvement in HIV testing percentages. In the PED setting, HIV testing might not be a priority, especially when resources are lacking or where there are competing priorities. While removal of written consent and pretest counseling has removed some of the policy barriers that were previously cited, there still remain some perceived barriers which are limiting uptake of routine testing. ${ }^{1}$ In addition, laboratory operations typically make laboratory turnaround time a significant factor in many PEDs. HIV testing typically is done as a "batch" test run periodically by the laboratory. It would be difficult to justify "stat" testing order for a relatively nonurgent test which is being done for screening. Consistently offering HIV testing to eligible patients and using opt-out methods in the PED can improve testing percentages. Younger age groups are more likely not to test due to their lowered perception of risk as demonstrated in our study; this is a cause for concern, because the low perception of risk may be counter to their actual risk. Program addressing risk perception which emphasizes safe health practices should be developed to reduce HIV transmission. Integration of HIV testing into ED has been explicated as difficult because many ED providers do not think that HIV testing is in alignment with the mission of emergency medicine. ${ }^{6} \mathrm{ED}$ integration concerns also compounds the recent American College of Emergency Physicians policy statement which positions that EDs HIV screening programs deliver the greatest public health impact when the following factors are present: local prevalence of HIV infection is $\geq 0.1 \%$; screening procedures are practical, feasible, and do not interfere with the primary acute care mission of emergency medicine; integration exists between the ED and the resources of the entire health care system; presence of policies and procedures that addresses patient confidentiality, informed consent (state dependent), provider training, opportunities for counseling, and linkage to care; available and adequate funding to meet the operational and personnel costs required for programs sustainability and all local and state requirements are met. ${ }^{7}$ We infer that we can improve testing rate (especially among persons aged $<18$ years) by integrating our prediction model into our clinical care workflow at the POC to supplement testing and promptly identifying patients likely to reject to test.

\section{Disclosure}

Dr Lisa Henry Reid reports personal fees from Simply Speaking Speakers Bureau, outside the submitted work. The authors report no other conflicts of interest in this work.

\section{References}

1. Burke RC, Sepkowitz KA, Bernstein KT, et al. Why don't physicians test for HIV? A review of the US literature. AIDS. 2007;21(12):1617-1624.

2. Corey L. CDC recommendations for opt-out HIV testing. JAMA. 2009;301(3):274-275276

3. Christopoulos KA, Weiser SD, Koester KA, et al. Understanding patient acceptance and refusal of HIV testing in the emergency department. $B M C$ Public Health. 2012;12:3.

4. Zheng MY, Suneja A, Chou AL, Arya M. Physician barriers to successful implementation of US Preventive Services Task Force routine HIV testing recommendations. J Int Assoc Provid AIDS Care. 2014;13(3):200-205.

5. Stefan MS, Blackwell JM, Crawford KM, et al. Patients' attitudes toward and factors predictive of human immunodeficiency virus testing of academic medical clinics. Am J Med Sci. 2010;340(4):264-267.

6. Arbelaez C, Wright EA, Losina E, et al. Emergency provider attitudes and barriers to universal HIV testing in the emergency department. $J$ Emerg Med. 2012;42(1):7-14.

7. American College of Emergency Physicians (ACEP). HIV testing and screening in the emergency department. Policy statement. Ann Emerg Med. 2014;64(5):563. 


\section{Publish your work in this journal}

HIV/AIDS - Research and Palliative Care is an international, peerreviewed open access journal focusing on advances in research in HIV, its clinical progression and management options including antiviral treatment, palliative care and public healthcare policies to control viral spread. The journal is included in PubMed. The manuscript man-

Submit your manuscript here: https://www.dovepress.com/hivaids---research-and-palliative-care-journal 